\title{
APPLICATION OF A TRANSPORTATION FLUX FOR DETERMINING QUALITATIVE INDICES
}

A method of using the transportation flux of a manufacturing system for determining its qualitative parameters has been presented. Those subjects were discussed in many papers [1 - 4], however the presented method may be used for a preliminary determination of functionality of manufacturing system parameters when, as a consequence of its modernization a change in equipment of a certain section or a replacement of individual devices was planned. Such actions cause a necessity to determine the system's operating efficiency and reliability after changing parameters as well as the impact of working processes on the product quality. Such actions were conducted as a result of performed research works no. KBN nr 8 T07 D 03421 [5].

\section{Transportation flux}

A change in qualitative (final) product parameters can be caused by:

1. A change in nominal data of working equipment. This situation can occur as a consequence of lacking periodical maintenance or a slow wear and tear of the equipment.

2. A supply of a defective workpiece (subassembly) to the working station causing the making of a defective product and under extreme circumstances a damage of a manufacturing tool or a failed action of the working station, e.g. as a result of impossibility to fix the workpiece at the working station. The incoming defected workpieces create a random flow of damage level, whose value, validated as a number of discards, is often estimated in course of production preparation. The damage level value is estimated under specific manufacturing duration time frames and is usually considered as a permissible damage level.

3. A damage of manufacturing tool. If the workpiece was good, we experience a random damage of a tool making impossible to fulfill its basic functions. The product is removed from the working station and the working tool must be replaced.

To conduct a random quality control of a mounted assembly (by sampling) a number of workpieces to be taken for control from among the total number of workpieces in the transportation flux must be determined. This enables determining the process quality by using the basic coefficients, assuming the following initial data: $N$ - total number of workpieces leaving the working station,

$N_{D}$ - total number of discarded workpieces

$N_{K}$ - total number of workpieces subject to control

$N_{D K}$ - total number of workpieces with defects found at the control

The level of discard (defected workpieces):

$$
k_{D}=\frac{N_{D}}{N}
$$

The mean value of discards within a certain time range determined as a results of conducted control processes:

$$
k_{D}=\frac{N_{D}-N_{D K}}{N-N_{K}}
$$

The coefficient of controlled assemblies:

$$
k_{K}=\frac{N_{K}}{N}
$$

The coefficient of discarded (defected) workpieces:

$$
k_{U}=\frac{N_{D K}}{N_{K}}
$$

The next step is to determine the optimum number of workpieces subject to control in order to determine the share of defected (discarded) products at the whole manufacturing lot. An important precaution is the uniformity and randomness of allocation of defected products among good ones. i.e. a gathering of defected products into clearly visible clusters inside the whole lot is unacceptable. The basic parameter to be determined in this case is $k_{E}$ - the share of defected products.

$N_{D}$ - number of defected parts

$N_{Q}$ - number of good quality parts.

The $\mathrm{kE}$ parameter was determined by means of a calculating program created on basis of the Statistic program. After choosing a lot (population) quantity a current share of defected products is determined as a ratio of actually known number of defected products to the number of controlled ones. An accuracy of analysis can be supplemented by indicating the initial (inaccurate calculation) and final (accurate calculation) point of stabilization. This is the point at the diagram (Fig. 1) determining such a number of controlled products after which the curve of quantity of defected products is stabilized. To find the versatile point of stabilization

\footnotetext{
* Aleksander Nieoczym

Technical University of Lublin, ul. Nadbystrzycka 36, 20-618 Lublin, Poland, E-mail: a.nieoczym@pollub.pl
} 


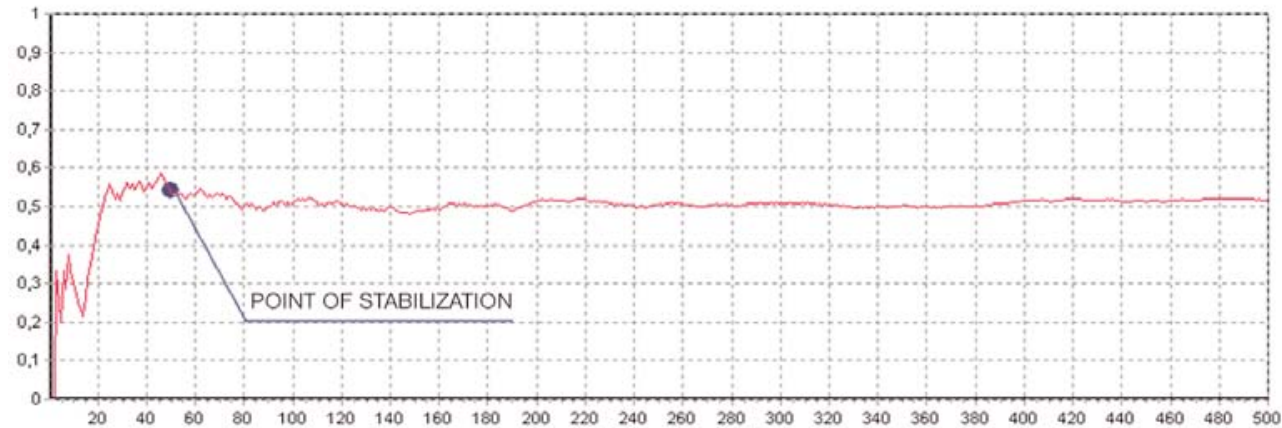

for a certain size of the lot, the program analyses several sets of equal size (the number of defected parts at each set is a random value), whereas the point of stabilization is an arithmetic mean of all points of tested sets of equal size.

\section{Process monitoring at the working station}

Sensors are used to control the manufacturing processes. The sensors are placed not only at the working station, where the main process is conducted but also at devices being components of tooling e.g. supplying or fixing devices. In many cases they are flexible working stations able to process workpieces of various geometric forms and various numbers of treatment cycles. Therefore, not in each case it is needed to acquire data from all measurement sensors. At every case the number of active sensors, i.e. the number of process parameters and variables to be read out The $S$ parameters (states) are determined and divided into subsets $S_{n}$ containing qualitatively identical parameters corresponding to treatment operations at working stations. The $S$ parameters correspond also with $L_{m}$ parameters aligned by quality and measured at the tooling. The $S$ states are e.g. process speed, process time, the $L$ parameters are e.g. position of supplying device's arm, speed of supplying workpieces into a working station.

To conduct the general control of work, it is enough to read out parameters of randomly selected $S_{n}$ or $L_{m}$ subsets. This enables determining the place of arising of irregularities within the group of parts read out by one of $S$ or $L$ subsets. In case of full control all $S$ and $L$ states are used. Let $T$ stand for the quantity of measured parameters:

$$
T=\left(S_{n}\left\lceil L_{m}\right), \quad n=(1 \ldots N), \quad m=(1 \ldots M)\right.
$$

Two extreme cases can be obtained, if $n=1$ and $\mathrm{m}=\mathrm{M}$, then $T=M+1$, i.e. the control gets parametric. If only the work readiness is controlled, only one signal $S_{n}(n+1)$ is obtained, but to find the irregularities, all $m=M$ parameters must be tested. The second extreme case is analogous. At constant $L_{m}$ all $S_{n}(n=N)$ parameters are read out. Having assumed: $n=m=N$, in the first case the area of investigation is: $A=n_{m}=N$, the number of measured parameters: $T=n+m=1+N$. For the second case: $A=N^{2}$.

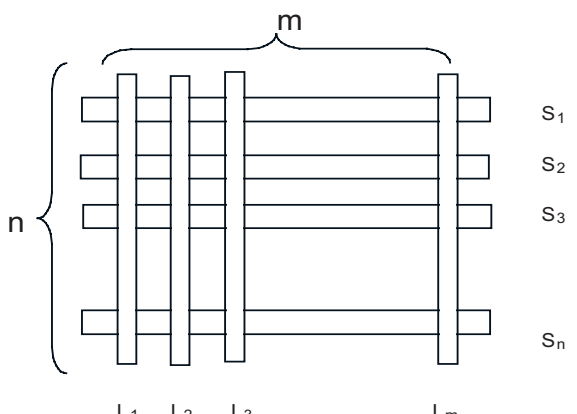

Fig. 2. Graphic presentation of relationship between parameters of the working process states and parameters of tooling [6].

S1 ... Sn - parameters, states corresponding to a certain process operation at the working station, $\mathrm{L} 1$... Lm - parameters measured at the tooling, $\mathrm{A}=\mathrm{mn}=\mathrm{N}$ - the whole area of investigation.

\section{References}

[1] CHRYSSOLOURIS, G.: Manufacturing systems, Theory and practice, Springer - Verlang, New York 1992.

[2] DASZCZIENKO, A., BIEŁOUSOW, A.: Design of automated line (in Russian). High School, Moscow, 1986.

[3] DRUŻININ, G.: About evaluation reliability technological systems with loading devices (in Russian). Standardization and Quality, $\mathrm{n} 10$, pp 8 - 12, 1994.

[4] KUMAR, V., WANG, S.: Reliability enhancement by time and space redundancy in multistage interconnection networks, IEEE Tranis. Reliab., vol 40, n 4, pp. 461 - 473, 1991

[5] NIEOCZYM, A.: Problems designing hierarchical organized assembly systems (in Polish). LTN, Lublin, 2002.

[6] Fundamental problems of reliability in theory and practice (in Russian). Moscow, 1975. 